\title{
CLUSTERING PHENOMENA FOR LINEAR PERTURBATION OF THE YAMABE EQUATION
}

\author{
ANGELA PISTOIA AND GIUSI VAIRA
}

This paper is warmly dedicated to Professor Abbas Bahri on the occasion of his 60th birthday

\begin{abstract}
Let $(M, g)$ be a non-locally conformally flat compact Riemannian manifold with dimension $N \geq 7$. We are interested in finding positive solutions to the linear perturbation of the Yamabe problem

$$
-\mathcal{L}_{g} u+\epsilon u=u^{\frac{N+2}{N-2}} \text { in }(M, g)
$$

where the first eigenvalue of the conformal laplacian $-\mathcal{L}_{g}$ is positive and $\epsilon$ is a small positive parameter. We prove that for any point $\xi_{0} \in M$ which is non-degenerate and non-vanishing minimum point of the Weyl's tensor and for any integer $k$ there exists a family of solutions developing $k$ peaks collapsing at $\xi_{0}$ as $\epsilon$ goes to zero. In particular, $\xi_{0}$ is a non-isolated blow-up point.
\end{abstract}

Keywords: Yamabe problem, linear perturbation, blow-up points

AMS subject classification: 35J35, 35J60

\section{INTRODUCTION}

Let $(M, g)$ be a smooth, compact Riemannian manifold of dimension $N \geq 3$. The Yamabe problem consists in finding metrics of constant scalar curvature in the conformal class of $g$. It is equivalent to finding a positive solution to the problem

$$
\mathcal{L}_{g} u+\kappa u^{\frac{N+2}{N-2}}=0 \text { in } M,
$$

for some constant $\kappa$. Here $\mathcal{L}_{g} u:=\Delta_{g} u+\frac{N-2}{4(N-1)} R_{g} u$ is the conformal laplacian, $\Delta_{g}$ is the Laplace-Beltrami operator and $R_{g}$ is the scalar curvature of the manifold .

In particular, if $u$ solves (1.1), then the scalar curvature of the metric $\tilde{g}=u^{\frac{4}{N-2}} g$ is nothing but $\frac{4(N-1)}{N-2} \kappa$. Yamabe problem has been completely solved by Yamabe [26], Aubin [1], Trudinger [25] and Schoen [20] (see also the proof given by Bahri [2]). The solution is unique in the case of negative scalar curvature and it is unique (up to a constant factor) in the case of zero scalar curvature. The uniqueness is not true anymore in the case of positive scalar curvature. Indeed, Schoen [21] and Pollack in [16] exhibit examples where a large number of high energy solutions with high Morse index exist. Thus it is natural to ask if the set of solutions is compact or not as it was raised by Schoen in [22]. It is also useful to point out that in the case of the round sphere $\left(\mathbb{S}^{N}, g_{0}\right)$ the compactness does not hold (see Obata in [15]). Indeed, the scalar curvature $R_{g_{0}}=N(N-1)$ and the Yamabe problem (1.1) reads as

$$
-\Delta_{g_{0}} u+\frac{N(N-2)}{4} u=u^{\frac{N+2}{N-2}} \text { in }\left(\mathbb{S}^{N}, g_{0}\right)
$$

which is equivalent (via the stereographic projection) to the equation in the Euclidean space

$$
-\Delta U=U^{\frac{N+2}{N-2}} \text { in } \mathbb{R}^{N} \text {. }
$$

It is known that (1.2) has infinitely many solutions, the so called standard bubbles,

$$
U_{\mu, y}(x)=\mu^{-\frac{N-2}{2}} U\left(\frac{x-y}{\mu}\right), x, y \in \mathbb{R}^{N}, \mu>0, \text { where } U(x):=\alpha_{N} \frac{1}{\left(1+|x|^{2}\right)^{\frac{N-2}{2}}} .
$$

Here $\alpha_{N}:=N(N-2)^{\frac{N-2}{4}}$.

The compactness turns out to be true when the dimension of the manifold satisfies $3 \leq N \leq 24$ as it was shown by Khuri, Marques and Schoen [9]) (previous results were obtained by Schoen [23], Schoen and Zhang [24], Li and Zhu [12], Li and Zhang [11], Marques [13] and Druet [6]), while it is false when $N \geq 25$ thanks to the examples built by Brendle [4] and Brendle and Marques [5]. The proof of compactness strongly relies on proving sharp pointwise estimates at a blow-up point of the solution. In particular, when compactness holds every sequence of unbounded solutions to (1.1) must blow-up at some points of 
the manifold which are necessarily isolated and simple, i.e. around each blow-up point $\xi_{0}$ the solution can be approximated by a standard bubble (see (1.3))

$$
u_{n}(x) \sim \alpha_{N} \frac{\mu_{n}^{\frac{N-2}{2}}}{\left(\mu_{n}^{2}+\left(d_{g}\left(x, \xi_{n}\right)\right)^{2}\right)^{\frac{N-2}{2}}} \text { for some } \xi_{n} \rightarrow \xi_{0} \text { and } \mu_{n} \rightarrow 0 .
$$

More precisely, let $u_{n}$ be a sequence of solutions to problem (1.1). We say that $u_{n}$ blows-up at a point $\xi_{0} \in M$ if there exists $\xi_{n} \in M$ such that $\xi_{n} \rightarrow \xi_{0}$ and $u_{n}\left(\xi_{n}\right) \rightarrow+\infty$. $\xi_{0}$ is said to be a blow-up point for $u_{n}$. Blow-up points can be classified according to the definitions introduced by Schoen in [22]. $\xi_{0} \in M$ is an isolated blow-up point for $u_{n}$ if there exists $\xi_{n} \in M$ such that $\xi_{n}$ is a local maximum of $u_{n}, \xi_{n} \rightarrow \xi_{0}$, $u_{n}\left(\xi_{n}\right) \rightarrow+\infty$ and there exist $c>0$ and $R>0$ such that

$$
0<u_{n}(x) \leq c \frac{1}{d_{g}\left(x, \xi_{n}\right)^{\frac{N-2}{2}}} \text { for any } x \in B\left(\xi_{0}, R\right) .
$$

Moreover, $\xi_{0} \in M$ is an isolated simple blow-up point for $u_{n}$ if the function

$$
\hat{u}_{n}(r):=r^{\frac{N-2}{2}} \frac{1}{\left|\partial B\left(\xi_{n}, r\right)\right|_{g}} \int_{\partial B\left(\xi_{n}, r\right)} u_{n} d \sigma_{g}
$$

has a exactly one critical point in $(0, R)$.

Motivated by the previous consideration, we are led to study the linear perturbation of the Yamabe problem

$$
-\mathcal{L}_{g} u+\epsilon u=u^{\frac{N+2}{N-2}}, u>0, \text { in }(M, g)
$$

where the first eigenvalue of $-\mathcal{L}_{g}$ is positive and $\epsilon$ is a small parameter. In particular, we address the following questions.

(i) Do there exist solutions to (1.4) which blow-up as $\epsilon \rightarrow 0$ ?

(ii) Do there exist solutions to (1.4) with non-isolated blow-up points, namely with clustering blow-up points?

(iii) Do there exist solutions to (1.4) with non-isolated simple blow-up points, namely with towering blow-up points?

Concerning question (i), Druet in [6] proved that equation (1.4) does not have any blowing-up solution when $\epsilon<0$ and $N=3,4,5$ (except when the manifold is conformally equivalent to the round sphere). It is completely open the case when the dimension is $N \geq 6$. The situation is completely different when $\epsilon>0$. Indeed, if $N=3$ no blowing-up solutions exist as proved by Li-Zhu [12], while if $m \geq 4$ blowing-up solutions do exist as shown by Esposito, Pistoia and Vetois in [8]. In particular, if the dimension $N \geq 6$ and the manifold is not locally conformally flat, Esposito, Pistoia and Vetois built solutions which blow-up at non-vanishing stable critical points $\xi_{0}$ of the Weyl's tensor, i.e. $\mid$ Weyl $\left.g_{g}\left(\xi_{0}\right)\right|_{g} \neq 0$. In this paper, we show that the blowing-up point $\xi_{0}$ is not-isolated as soon as it is a non-degenerate minimum point of the Weyl's tensor. This result gives a positive answer to question (ii). Finally, a positive answer to question (iii) has been giving by Morabito, Pistoia and Vaira in a forthcoming paper [14].

Now, let us state the main result obtained in this paper.

Theorem 1.1. Let $(M, g)$ be not locally conformally flat and $N \geq 7$. Let $\xi_{0} \in M$ be a non-degenerate minimum point of $\xi \rightarrow\left|\operatorname{Weyl}_{g}(\xi)\right|_{g}^{2}$. Then, for any $k \in \mathbb{N}$, there exist $\xi_{\varepsilon}^{j} \in M$ for $j=1, \ldots, k$ and $\varepsilon_{k}>0$ such that for all $\varepsilon \in\left(0, \varepsilon_{k}\right)$ the problem (1.4) has a solution $\left(u_{\varepsilon}\right)_{\varepsilon}$ with $k$ positive peaks at $\xi_{\varepsilon}^{j}$ and $\xi_{\varepsilon}^{j} \rightarrow \xi_{0}$ as $\varepsilon \rightarrow 0$.

Let us point out that Robert and Vétois in [18] built solutions having clustering blow-up points for a special class of perturbed Yamabe type equations which look like

$$
-\mathcal{L}_{g} u+\epsilon H u=u^{\frac{N+2}{N-2}}, u>0, \text { in }(M, g) .
$$

where the potential $H$ is chosen with $k$ distinct strict local maxima concentrating at a point $\xi_{0}$ with $\left|\operatorname{Weyl}_{g}\left(\xi_{0}\right)\right|_{g} \neq 0$. Indeed, these maxima points generate solutions with $k$ positive peaks collapsing to $\xi_{0}$ as $\epsilon$ goes to zero. Their result is related to a suitable choice of the potential $H$, but actually our result shows that the clustering phenomena is intrinsic in the geometry of the manifold. 
Let us give an example. The warped product $\left(S^{n} \times S^{m}, g_{S^{n}} \otimes f^{2} g_{S^{m}}\right)$, is the Riemannian manifold $S^{n} \times S^{m}$ equipped with the metric $g=g_{S^{n}} \otimes f^{2} g_{S^{m}}$. Here $f: S^{n} \rightarrow \mathrm{R}$ is a positive function called warping function. It is easy to see that if the warping function $f \equiv 1$ than the the product manifold $\left(S^{n} \times S^{m}, g_{S^{n}} \otimes g_{S^{m}}\right)$ has Weyl tensor different from zero at any point. Using similar argoments to the ones used in [17], we can prove that for generic warping functions $f$ close to the constant 1 , the Weyl tensor has a non-degenerate and non-vanishing minimum point.

The proof of our result relies on a finite dimensional Ljapunov-Schmidt reduction, whose main steps are described in Section 3 and their proofs are postponed in Section 4. Section 2 is devoted to recall some known results.

\section{Preliminaries}

We provide the Sobolev space $H_{g}^{1}(M)$ with the scalar product

$$
\langle u, v\rangle=\int_{M}\langle\nabla u, \nabla v\rangle_{g} d \nu_{g}+\beta_{N} \int_{M} \mathrm{R}_{g} u v d \nu_{g}
$$

where $d \nu_{g}$ is the volume element of the manifold. Here $\beta_{N}:=\frac{N-2}{4(N-1)}$. We let $\|\cdot\|$ be the norm induced by $\langle\cdot, \cdot\rangle$. Moreover, for any function $u$ in $L^{q}(M)$, we denote the $L^{q}$-norm of $u$ by $\|u\|_{q}=\left(\int_{M}|u|^{q} d \nu_{g}\right)^{1 / q}$.

We let $1^{*}: L^{\frac{2 N}{N+2}}(M) \rightarrow H_{g}^{1}(M)$ be the adjoint operator of the embedding $1: H_{g}^{1}(M) \hookrightarrow L^{2^{*}}(M)$, i.e. for any $w$ in $L^{\frac{2 N}{N+2}}(M)$, the function $u=1^{*}(w)$ in $H_{g}^{1}(M)$ is the unique solution of the equation $-\Delta_{g} u+\beta_{N} \mathrm{R}_{g} u=w$ in $M$. By the continuity of the embedding of $H_{g}^{1}(M)$ into $L^{2^{*}}(M)$, we get

$$
\left\|1^{*}(w)\right\| \leq C\|w\|_{\frac{2 N}{N+2}}
$$

for some positive constant $C$ independent of $w$. We rewrite problem (1.4) as

$$
u=1^{*}[f(u)-\varepsilon u], \quad u \in \mathrm{H}_{g}^{1}(M)
$$

where we set $f(u):=\left(u^{+}\right)^{p}$ with $p=\frac{N+2}{N-2}$.

We also define the energy $J_{\epsilon}: H_{g}^{1}(M) \rightarrow \mathbb{R}$

$$
J_{\epsilon}(u):=\frac{1}{2} \int_{M}\left(\left|\nabla_{g} u\right|^{2}+\beta_{N} \mathrm{R}_{g} u^{2}+\epsilon u^{2}\right) d \nu_{g}-\frac{1}{p+1} \int_{M}\left(u^{+}\right)^{p+1} d \nu_{g},
$$

whose critical points are solutions to the problem (1.4).

We are going to read the euclidean bubble defined in (1.3) on the manifold via a geodesic normal coordinate system around a point $\xi \in M$, i.e.

$$
\mathcal{U}_{\mu, \xi}(z)=U_{\mu, 0}\left(\exp _{\xi}^{-1}(z)\right)=\mu^{-\frac{N-2}{2}} U\left(\frac{\exp _{\xi}^{-1}(z)}{\mu}\right), z \in B_{g}(\xi, r) .
$$

It is necessary to write the conformal laplacian in geodesic normal coordinates around the point $\xi$. In particular, if $x \in B(0, r)$ using standard properties of the exponential map we can write

$$
-\Delta_{g} u=-\Delta u-\left(g^{i j}-\delta^{i j}\right) \partial_{i j}^{2} u+g^{i j} \Gamma_{i j}^{k} \partial_{k} u,
$$

with

$$
g^{i j}(x)=\delta^{i j}(x)-\frac{1}{3} R_{i a b j}(\xi) x_{a} x_{b}+O\left(|x|^{3}\right) \text { and } g^{i j}(x) \Gamma_{i j}^{k}(x)=\partial_{l} \Gamma_{i i}^{k}(\xi) x_{l}+O\left(|x|^{2}\right) .
$$

Here $R_{i a b j}$ denotes the Riemann curvature tensor and $\Gamma_{i i}^{k}$ the Christoffel's symbols. Therefore, if we compare the conformal laplacian with the euclidean laplacian of the bubble the error at main order looks like

$$
\mathcal{L}_{g} \mathcal{U}_{\mu, \xi}-\Delta \mathcal{U}_{\mu, \xi} \sim-\frac{1}{3} \sum_{a, b, i, j=1}^{N} R_{i a b j}(\xi) x_{a} x_{b} \partial_{i j}^{2} U_{\mu, 0}+\sum_{i, l, k=1}^{N} \partial_{l} \Gamma_{i i}^{k}(\xi) x_{l} \partial_{k} U_{\mu, 0}+\beta_{N} R_{g}(\xi) U_{\mu, 0} .
$$

For later purposes, it is necessary to kill this main term by adding to the bubble an higher order term $V$ which is defined as follows. First, we remind that any solution of the linear equation (see [3])

$$
-\Delta v=p U^{p-1} v \quad \text { in } \mathbb{R}^{N}
$$


is a linear combination of the functions

$$
\psi^{0}(x)=x \cdot \nabla U(x)+\frac{N-2}{2} U(x) \text { and } \psi^{i}(x)=\partial_{i} U(x), i=1, \ldots, N .
$$

Next, we introduce the higher order term $V$ which has been built in Section 2 in [7].

Proposition 2.1. For any point $\xi \in M$, there exist $\nu(\xi) \in \mathbb{R}$ and a function $V \in \mathcal{D}^{1,2}\left(\mathbb{R}^{N}\right)$ solution to

$$
-\Delta V-f^{\prime}(U) V=-\sum_{a, b, i, j=1}^{N} \frac{1}{3} R_{i a b j}(\xi) x_{a} x_{b} \partial_{i j}^{2} U+\sum_{i, l, k=1}^{N} \partial_{l} \Gamma_{i i}^{k}(\xi) x_{l} \partial_{k} U+\beta_{N} \mathrm{R}_{g}(\xi) U+\nu(\xi) \psi^{0} \text { in } \mathbb{R}^{N},
$$

with

$$
\int_{\mathbb{R}^{N}} V(x) \psi^{i}(x) d x=0, i=0,1, \ldots, N .
$$

Moreover, there exists $C \in \mathbb{R}$ such that

$$
|V(x)|+|x|\left|\partial_{k} V(x)\right|+|x|^{2}\left|\partial_{i j}^{2} V(x)\right| \leq C \frac{1}{\left(1+|x|^{2}\right)^{\frac{N-4}{2}}}, \quad x \in \mathbb{R}^{N} .
$$

\section{Clustering}

3.1. The ansatz: the cluster. Let $r_{0}$ be a positive real number less than the injectivity radius of $M$ and $\chi$ be a smooth cut-off function such that $0 \leq \chi \leq 1$ in $\mathbb{R}, \chi \equiv 1$ in $\left[-r_{0} / 2, r_{0} / 2\right]$, and $\chi \equiv 0$ out of $\left[-r_{0}, r_{0}\right]$. Let also $\eta$ be a smooth cutoff function such that $0 \leq \eta \leq 1$ in $\mathbb{R}, \eta \equiv 1$ in $[-1,1]$, and $\eta \equiv 0$ out of $[-2,2]$.

Let $k \geq 1$ be a fixed integer. Assume that $\xi_{0} \in M$ is a non degenerate minimum point of $\xi \rightarrow$ $\mid$ Weyl $\left._{g}(\xi)\right|_{g} ^{2}$ with $\mid$ Weyl $_{g}\left(\xi_{0}\right) \mid \neq 0$, i.e.

$$
\nabla_{g} \mid \text { Weyl }\left._{g}\left(\xi_{0}\right)\right|_{g} ^{2}=0 \text { and the quadratic form } \mathcal{Q}\left(\xi_{0}\right):=D_{g}^{2} \mid \text { Weyl }\left._{g}\left(\xi_{0}\right)\right|_{g} ^{2} \text { is positive definite. }
$$

Set

$$
d_{0}:=\left(\frac{B_{N}}{2 A_{N} \mid \text { Weyl }\left._{g}\left(\xi_{0}\right)\right|_{g} ^{2}}\right)^{1 / 2} \quad\left(A_{N} \text { and } B_{N}\right. \text { are positive constants defined in (4.3)) }
$$

and let us choose

and for any $i=1, \ldots, k$

$$
\tau_{1}, \ldots, \tau_{k} \in \mathbb{R}^{N} \text { with } \tau_{i} \neq \tau_{j} \text { if } i \neq j
$$

$$
\mu_{i}=\varepsilon^{\alpha}\left(d_{0}+d_{i} \varepsilon^{\beta}\right), \text { where } d_{1}, \ldots, d_{k} \in(0,+\infty), \alpha:=\frac{1}{2}, \beta:=\frac{N-6}{2 N} .
$$

Then, let us define

$$
\begin{aligned}
\mathcal{W}_{i}(z):= & \chi\left(d_{g}\left(z, \xi_{0}\right)\right) \mu_{i}^{-\frac{N-2}{2}} U\left(\frac{\exp _{\xi_{0}}^{-1}(z)-\varepsilon^{\beta} \tau_{i}}{\mu_{i}}\right) \\
& +\mu_{i}^{2} \eta\left(\frac{\left|\exp _{\xi_{0}}^{-1}(z)-\varepsilon^{\beta} \tau_{i}\right|}{\mu_{i}}\right) \chi\left(d_{g}\left(z, \xi_{0}\right)\right) \mu_{i}^{-\frac{N-2}{2}} V\left(\frac{\exp _{\xi_{0}}^{-1}(z)-\varepsilon^{\beta} \tau_{i}}{\mu_{i}}\right), z \in M
\end{aligned}
$$

where the functions $U$ and $V$ are defined, respectively, in (1.3) and (2.9). Set

$$
\mathcal{C}:=\left\{\left(\tau_{1}, \ldots, \tau_{k}\right) \in \mathbb{R}^{k N}: \tau_{i} \neq \tau_{j} \text { if } i \neq j\right\} .
$$

We look for solutions of equation (1.4) or (2.3) of the form

$$
u_{\varepsilon}(z)=\sum_{i=1}^{k} \mathcal{W}_{i}(z)+\phi_{\varepsilon}(z),
$$

where the remainder term $\phi_{\varepsilon}$ belongs to the space $\mathcal{K}^{\perp}$ defined as follows. For any $i=1, \ldots, k$ we introduce the functions

$$
Z_{j, i}(z)=\chi\left(d_{g}\left(z, \xi_{0}\right)\right) \mu_{i}^{-\frac{N-2}{2}} \psi^{j}\left(\frac{\exp _{\xi_{0}}^{-1}(z)-\varepsilon^{\beta} \tau_{i}}{\mu_{i}}\right), j=0,1, \ldots, N
$$


where the functions $\psi^{j}$ are defined in (2.8). We define the subspaces

$$
\mathcal{K}:=\operatorname{Span}\left\{1^{*}\left(Z_{j, i}\right), j=0,1, \ldots, N, i=1, \ldots, k\right\}
$$

and

$$
\mathcal{K}^{\perp}:=\left\{\phi \in H_{g}^{1}(M):\left\langle\phi, 1^{*}\left(Z_{j, i}\right)\right\rangle=0, \quad j=0, \ldots, N, i=1, \ldots, k\right\}
$$

and we also define the projections $\Pi$ and $\Pi^{\perp}$ of $H_{g}^{1}(M)$ onto $\mathcal{K}$ and $\mathcal{K}^{\perp}$, respectively.

Therefore, equation 2.3 turns out to be equivalent to the system

$$
\begin{aligned}
& \Pi^{\perp}\left\{u_{\varepsilon}-1^{*}\left[f\left(u_{\varepsilon}\right)-\varepsilon u_{\varepsilon}\right]\right\}=0, \\
& \Pi\left\{u_{\varepsilon}-1^{*}\left[f\left(u_{\varepsilon}\right)-\varepsilon u_{\varepsilon}\right]\right\}=0 .
\end{aligned}
$$

where $u_{\varepsilon}$ is given in (3.6).

3.2. The remainder term: solving the equation (3.8). In order to find the remainder term $\phi_{\varepsilon}$ we rewrite $(3.8)$ as

$$
\mathcal{E}+\mathcal{L}\left(\phi_{\varepsilon}\right)+\mathcal{N}\left(\phi_{\varepsilon}\right)=0
$$

where the error term $\mathcal{E}$ is defined by

$$
\mathcal{E}:=\Pi^{\perp}\left\{\sum_{i=1}^{k} \mathcal{W}_{i}-1^{*}\left[f\left(\sum_{i=1}^{k} \mathcal{W}_{i}\right)-\varepsilon \sum_{i=1}^{k} \mathcal{W}_{i}\right]\right\}
$$

the linear operator $\mathcal{L}$ is defined by

$$
\mathcal{L}\left(\phi_{\varepsilon}\right):=\Pi^{\perp}\left\{\phi_{\varepsilon}-1^{*}\left[f^{\prime}\left(\sum_{i=1}^{k} \mathcal{W}_{i}\right) \phi_{\varepsilon}-\varepsilon \phi_{\varepsilon}\right]\right\}
$$

and the higher order term $\mathcal{N}$ is defined by

$$
\mathcal{N}:=\Pi^{\perp}\left\{-1^{*}\left[f\left(\sum_{i=1}^{k} \mathcal{W}_{i}+\phi_{\varepsilon}\right)-f\left(\sum_{i=1}^{k} \mathcal{W}_{i}\right)-f^{\prime}\left(\sum_{i=1}^{k} \mathcal{W}_{i}\right) \phi_{\varepsilon}\right]\right\} .
$$

In order to solve equation (3.8), first of all we need to evaluate the $H_{g}^{1}(M)$ - norm of the error term $\mathcal{E}$. This is done in the following lemma whose proof is postponed in Section 4.

Lemma 3.1. For any compact subset $A \subset(0,+\infty)^{k} \times \mathcal{C}$ there exists a positive constant $C$ and $\varepsilon_{0}>0$ such that for any $\left(d_{1}, \ldots, d_{k}, \tau_{1}, \ldots, \tau_{k}\right) \in A$ and for any $\varepsilon \in\left(0, \varepsilon_{0}\right)$ it holds

$$
\|\mathcal{E}\| \leq C \begin{cases}\varepsilon^{\frac{5}{4}} & \text { if } N=7 \\ \varepsilon^{\frac{3}{2}}|\ln \varepsilon|^{\frac{5}{8}} & \text { if } N=8 \\ \varepsilon^{\frac{3}{2}} & \text { if } N \geq 9 .\end{cases}
$$

Next, we need to understand the invertibility of the linear operators $\mathcal{L}$. This is done in the following lemma whose proof can be carried out as in [19].

Lemma 3.2. For any compact subset $A \subset(0,+\infty)^{k} \times \mathcal{C}$ there exists a positive constant $C$ and $\varepsilon_{0}>0$ such that for any $\left(d_{1}, \ldots, d_{k}, \tau_{1}, \ldots, \tau_{k}\right) \in A$ and for any $\varepsilon \in\left(0, \varepsilon_{0}\right)$ it holds

$$
\|\mathcal{L}(\phi)\| \geq C\|\phi\| \text { for any } \phi \in \mathcal{K}^{\perp} .
$$

Finally, we are able to solve equation (3.8). This is done in the following proposition, whose proof is postponed in Section 4 and relies on a standard contraction mapping argument.

Proposition 3.1. For any compact subset $A \subset(0,+\infty)^{k} \times \mathcal{C}$ there exists a positive constant $C$ and $\varepsilon_{0}$ such that for $\varepsilon \in\left(0, \varepsilon_{0}\right)$ and for any $\left(d_{1}, \ldots, d_{k}, \tau_{1}, \ldots, \tau_{k}\right) \in A$ there exists a unique function $\phi_{\varepsilon} \in \mathcal{K}^{\perp}$ which solves equation (3.8) such that

$$
\left\|\phi_{\varepsilon}\right\| \leq C \varepsilon^{\frac{3(N-2)}{2 N}+\zeta}
$$

for some $\zeta>0$. Moreover, the map $\left(d_{1}, \ldots, d_{\ell}, \tau_{1}, \ldots, \tau_{k}\right) \rightarrow \phi_{\ell, \varepsilon}\left(d_{1}, \ldots, d_{\ell}, \tau_{1}, \ldots, \tau_{k}\right)$ is of class $C^{1}$ and

$$
\left\|\nabla_{\left(d_{1}, \ldots, d_{\ell}, \tau_{1}, \ldots, \tau_{k}\right)} \phi_{\varepsilon}\right\| \leq C \varepsilon^{\frac{3(N-2)}{2 N}+\zeta}
$$

for some positive constants $C$ and $\zeta$. 
3.3. The reduced problem: proof of Theorem 1.1. Let us introduce the reduced energy, defined by

$$
\widetilde{J}_{\varepsilon}\left(d_{1}, \ldots, d_{k}, \tau_{1}, \ldots, \tau_{k}\right):=J_{\varepsilon}\left(\sum_{i=1}^{k} \mathcal{W}_{i}+\phi_{\varepsilon}\right),\left(d_{1}, \ldots, d_{k}, \tau_{1}, \ldots, \tau_{k}\right) \in(0,+\infty)^{k} \times\left(\mathbb{R}^{N}\right)^{k}
$$

where the remainder term $\phi_{\varepsilon}$ is defined in Proposition 3.1.

The following result allows as usual to reduce our problem to a finite dimensional one. The proof is standard and it is postponed in Section 4.

Proposition 3.2. (i) $\sum_{i=1}^{k} \mathcal{W}_{i}+\phi_{\varepsilon}$ is a solution to $(1.4)$ if and only if $\left(d_{1}, \ldots, d_{k}, \tau_{1}, \ldots, \tau_{k}\right) \in(0,+\infty)^{k} \times$ $\left(\mathbb{R}^{N}\right)^{k}$ is a critical point of the reduced energy (3.16)

(ii) The following expansion holds true

$$
\widetilde{J}_{\varepsilon}\left(d_{1}, \ldots, d_{k}, \tau_{1}, \ldots, \tau_{k}\right):=k D_{N}+c\left(\xi_{0}\right) \varepsilon^{2}+\varepsilon^{3 \frac{N-2}{N}} \mathfrak{J}\left(d_{1}, \ldots, d_{k}, \tau_{1}, \ldots, \tau_{k}\right)+o\left(\varepsilon^{3 \frac{N-2}{N}}\right)
$$

as $\varepsilon \rightarrow 0, C^{0}-$ uniformly with respect to $\left(d_{1}, \ldots, d_{k}, \tau_{1}, \ldots, \tau_{k}\right)$ in compact subsets of $(0,+\infty)^{k} \times \mathcal{C}$. Here $c\left(\xi_{0}\right):=k\left[-A_{N} \mid\right.$ Weyl $\left.\left._{g}\left(\xi_{0}\right)\right|_{g} ^{2} d_{0}^{4}+B_{N} d_{0}^{2}\right], A_{N}, B_{N}, D_{N}$ and $E_{N}$ are positive constants defined in (4.3) and

$$
\mathfrak{J}\left(d_{1}, \ldots, d_{k}, \tau_{1}, \ldots, \tau_{k}\right):=-\frac{1}{2} A_{N} d_{0}^{4} \sum_{i=1}^{k} \mathcal{Q}\left(\xi_{0}\right)\left(\tau_{i}, \tau_{i}\right)-E_{N} d_{0}^{N-2} \sum_{\substack{i, j=1 \\ i \neq j}}^{k} \frac{1}{\left|\tau_{i}-\tau_{j}\right|^{N-2}}-B_{N} \sum_{i=1}^{k} d_{i}^{2} .
$$

Proof of Theorem 1.1. By (i) of Proposition (3.2), it is sufficient to find a critical point of the reduced energy $\widetilde{J}_{\varepsilon}$. Now, the function $\mathfrak{J}$ defined in (3.18), has a maximum point which is stable under $C^{0}$-perturbations. Therefore, by (ii) of Proposition (3.2), we deduce that if $\varepsilon$ is small enough there exists $\left(d_{1 \varepsilon}, \ldots, d_{k \varepsilon}, \tau_{1 \varepsilon}, \ldots, \tau_{k \varepsilon}\right)$ critical point of $\widetilde{J}_{\varepsilon}$. That concludes the proof.

\section{Appendix}

For any $i=1, \ldots, k$, we set

$$
W_{i}(x):=\mu_{i}^{-\frac{N-2}{2}} U\left(\frac{x-\varepsilon^{\beta} \tau_{i}}{\mu_{i}}\right)+\eta\left(\frac{\left|x-\varepsilon^{\beta} \tau_{i}\right|}{\mu_{i}}\right) \chi\left(d_{g}\left(z, \xi_{0}\right)\right) \mu_{i}^{-\frac{N-6}{2}} V\left(\frac{x-\varepsilon^{\beta} \tau_{i}}{\mu_{i}}\right), x \in \mathbb{R}^{N} .
$$

It is important to point out that there exists $c>0$ such that

$$
\left|W_{i}(x)\right| \leq c \frac{\mu_{i}^{\frac{N-2}{2}}}{\left|x-\varepsilon^{\beta} \tau_{i}\right|^{N-2}} \forall x \in \mathbb{R}^{N} .
$$

4.1. Proof of Lemma 3.1. It is easy to see that, $(\nu(\xi)$ is defined in $(2.9))$

$$
\|\mathcal{E}\| \leq c \sum_{i=1}^{k}\left|-\Delta_{g} \mathcal{W}_{i}+\left(\beta_{N} \mathrm{R}_{g}+\varepsilon\right) \mathcal{W}_{i}-\nu(\xi) Z_{0, i}-f\left(\mathcal{W}_{i}\right)\right|_{\frac{2 N}{N+2}}+c\left|f\left(\sum_{i=1}^{k} \mathcal{W}_{i}\right)-\sum_{i=1}^{k} f\left(\mathcal{W}_{i}\right)\right|_{\frac{2 N}{N+2}}
$$

Arguing exactly as in Lemma 3.1 of [7], we can estimate each term

$$
\left|-\Delta_{g} \mathcal{W}_{i}+\left(\beta_{N} \mathrm{R}_{g}+\varepsilon\right) \mathcal{W}_{i}-\nu(\xi) Z_{0, i}-f\left(\mathcal{W}_{i}\right)\right|_{\frac{2 N}{N+2}}=\left\{\begin{array}{l}
O\left(\varepsilon^{\frac{5}{4}}\right) \text { if } N=7, \\
O\left(\varepsilon^{\frac{3}{2}}|\ln \varepsilon|^{\frac{5}{8}}\right) \text { if } N=8 \\
O\left(\varepsilon^{\frac{3}{2}}\right) \text { if } N \geq 9 .
\end{array}\right.
$$

Next, we show that

$$
\left|f\left(\sum_{i=1}^{k} \mathcal{W}_{i}\right)-\sum_{i=1}^{k} f\left(\mathcal{W}_{i}\right)\right|_{\frac{2 N}{N+2}}=O\left(\varepsilon^{3}\right)
$$


Set for any $h=1, \ldots, k B_{h}:=B\left(\varepsilon^{\beta} \tau_{h}, \varepsilon^{\beta} \sigma / 2\right)$ where $\sigma>0$ and small enough. For $(3.3) B_{h} \subset B\left(0, r_{0}\right)$ and they are disjoint. We write

$$
\begin{aligned}
& \left|f\left(\sum_{i=1}^{k} \mathcal{W}_{i}\right)-\sum_{i=1}^{k} f\left(\mathcal{W}_{i}\right)\right|_{\frac{2 N}{N+2}} \leq c\left[\left.\int_{B\left(0, r_{0}\right)}\left(1-\chi^{p+1}(|x|)\right)|\cdots|\right|^{\frac{2 N}{N+2}}|g(x)|^{\frac{1}{2}} d x\right]^{\frac{N+2}{2 N}} \\
& +c\left[\int_{B\left(0, r_{0}\right) \backslash \cup_{h} B_{h}}|\cdots| \frac{2 N}{N+2}|g(x)|^{\frac{1}{2}} d x\right]^{\frac{N+2}{2 N}}+c \sum_{h=1}^{k}\left[\int_{B_{h}}|\cdots| \frac{2 N}{N+2}|g(x)|^{\frac{1}{2}} d x\right]^{\frac{N+2}{2 N}} \\
& \leq c \sum_{i=1}^{k}\left[\int_{B\left(0, r_{0}\right)}\left(1-\chi^{p+1}(|x|)\right)\left|W_{i}\right|^{\frac{2 N}{N-2}}|g(x)|^{\frac{1}{2}} d x\right]^{\frac{N+2}{2 N}}+c\left[\int_{B\left(0, r_{0}\right) \backslash \cup_{h} B_{h}}\left|W_{i}\right|^{\frac{2 N}{N-2}}|g(x)|^{\frac{1}{2}} d x\right]^{\frac{N+2}{2 N}} \\
& +c \sum_{h=1}^{k}\left[\int_{B_{h}}\left|W_{h}^{p-1} \sum_{i \neq h} W_{i}\right|^{\frac{2 N}{N+2}}|g(x)|^{\frac{1}{2}} d x\right]^{\frac{N+2}{2 N}}+c \sum_{h=1}^{k}\left[\int_{B_{h}}\left|\sum_{i \neq h} W_{i}\right|^{\frac{2 N}{N-2}}|g(x)|^{\frac{1}{2}} d x\right]^{\frac{N+2}{2 N}} .
\end{aligned}
$$

Let us estimate each term in the previous expression. We use (4.1).

$$
\begin{aligned}
& \sum_{i=1}^{k}\left[\int_{B\left(0, r_{0}\right)}\left(1-\chi^{p+1}(|x|)\right)\left|W_{i}\right|^{\frac{2 N}{N-2}}|g(x)|^{\frac{1}{2}} d x\right]^{\frac{N+2}{2 N}} \leq c \sum_{i=1}^{k}\left[\int_{\mathbb{R}^{N} \backslash B\left(0, r_{0}\right)} \frac{\mu_{i}^{N}}{\left|x-\varepsilon^{\beta} \tau_{i}\right|^{2 N}} d x\right]^{\frac{N+2}{2 N}} \\
& \leq c \sum_{i=1}^{k} \frac{\mu_{i}^{\frac{N+2}{2}}}{\varepsilon^{\beta \frac{N+2}{2}}}\left[\int_{\mathbb{R}^{N} \backslash B\left(0, r_{0} / \varepsilon^{\beta}\right)} \frac{1}{\left|y-\tau_{i}\right|^{2 N}} d y\right]^{\frac{N+2}{2 N}} \\
& \leq c \varepsilon^{(\alpha-\beta) \frac{N+2}{2}+\alpha \frac{N+2}{2}} \leq c \varepsilon^{3 \frac{N+2}{2 N}}, \\
& {\left[\int_{B\left(0, r_{0}\right) \backslash \cup_{h} B_{h}}\left|W_{i}\right|^{\frac{2 N}{N+2}}|g(x)|^{\frac{1}{2}} d x\right]^{\frac{N+2}{2 N}} \leq c \frac{\mu_{i}^{\frac{N+2}{2}}}{\varepsilon^{\beta \frac{N+2}{2}}}\left[\int_{B\left(0, r_{0} / \varepsilon^{\beta}\right) \backslash \cup_{h} B\left(\tau_{h}, \sigma / 2\right)} \frac{1}{\left|y-\tau_{i}\right|^{2 N}} d y\right]^{\frac{N+2}{2 N}}} \\
& \leq C \varepsilon^{(\alpha-\beta) \frac{N+2}{2}} \leq C \varepsilon^{3 \frac{N+2}{2 N}}, \\
& \sum_{h=1}^{k}\left[\int_{B_{h}}\left|W_{h}^{p-1} \sum_{i \neq h} W_{i}\right|^{\frac{2 N}{N+2}}|g(x)|^{\frac{1}{2}} d x\right]^{\frac{N+2}{2 N}} \leq c \sum_{h=1}^{k} \sum_{i \neq h}\left[\int_{B_{h}} \frac{\mu_{h}^{\frac{4 N}{N+2}}}{\left|x-\varepsilon^{\beta} \tau_{h}\right|^{\frac{8 N}{N+2}}} \frac{\mu_{i}^{\frac{N(N-2)}{N+2}}}{\left|x-\varepsilon^{\beta} \tau_{i}\right|^{\frac{2 N(N-2)}{N+2}}} d x\right]^{\frac{N+2}{2 N}} \\
& \leq c \sum_{h=1}^{k} \sum_{i \neq h} \mu_{h}^{2} \mu_{i}^{\frac{N-2}{2}}\left[\int_{B_{h}} \frac{1}{\left|x-\varepsilon^{\beta} \tau_{h}\right|^{\frac{8 N}{N+2}}} d x\right]^{\frac{N+2}{2 N}} \\
& \leq c \sum_{h=1}^{k} \sum_{i \neq h} \mu_{h}^{2} \mu_{i}^{\frac{N-2}{2}}\left[\int_{B\left(0, \varepsilon^{\beta} \sigma / 2\right)} \frac{1}{|y|^{\frac{8 N}{N+2}}} d y\right]^{\frac{N+2}{2 N}} \\
& \leq c \sum_{h=1}^{k} \sum_{i \neq h} \mu_{h}^{2} \mu_{i}^{\frac{N-2}{2}} \varepsilon^{\beta \frac{N-6}{2}} \leq c \varepsilon^{3 \frac{N+2}{2 N}}
\end{aligned}
$$

and

$$
\begin{aligned}
\sum_{h=1}^{k}\left[\int_{B_{h}}\left|\sum_{i \neq h} W_{i}\right|^{\frac{2 N}{N-2}}|g(x)|^{\frac{1}{2}} d x\right]^{\frac{N+2}{2 N}} & \leq c \sum_{h=1}^{k} \sum_{i \neq h}\left[\int_{B_{h}} \frac{\mu_{i}^{N}}{\left|x-\varepsilon^{\beta} \tau_{i}\right|^{2 N}} d x\right]^{\frac{N+2}{2 N}} \\
& \leq C \frac{\mu_{i}^{\frac{N+2}{2}}}{\varepsilon^{\beta \frac{N+2}{2}}}\left[\int_{B\left(\tau_{h}, \sigma / 2\right)} \frac{1}{\left|y-\tau_{i}\right|^{2 N}} d y\right]^{\frac{N+2}{2 N}} \leq c \varepsilon^{3 \frac{N+2}{2 N}} .
\end{aligned}
$$

4.2. Proof of Proposition 3.2. It is quite standard to prove that

$$
J_{\varepsilon}\left(\sum_{i=1}^{k} \mathcal{W}_{i}+\phi_{\varepsilon}\right)=J_{\varepsilon}\left(\sum_{i=1}^{k} \mathcal{W}_{i}\right)+\Theta
$$


$C^{0}$ - uniformly with respect to $\left(d_{1}, \ldots, d_{k}, \tau_{1}, \ldots, \tau_{k}\right)$ in compact subset of $(0,+\infty)^{k} \times \mathcal{C}$, where $\Theta$ is a smooth function such that $|\Theta|,|\nabla \Theta|=O\left(\varepsilon^{3 \frac{N-2}{N}+\zeta}\right)$ for some small $\zeta>0$. We shall prove that

$$
\begin{aligned}
& J_{\varepsilon}\left(\sum_{i=1}^{k} \mathcal{W}_{i}\right)=k D_{N}+k \varepsilon^{2}\left[-A_{N}\left|\operatorname{Weyl}_{g}\left(\xi_{0}\right)\right|_{g}^{2}+B_{N} d_{0}^{2}\right] \\
& +\varepsilon^{3 \frac{N-2}{N}}\left[-\frac{1}{2} A_{N} d_{0}^{4} \sum_{i=1}^{k} \mathcal{Q}\left(\xi_{0}\right)\left(\tau_{i}, \tau_{i}\right)-E_{N} d_{0}^{N-2} \sum_{\substack{i, j=1 \\
i \neq j}}^{k} \frac{1}{\left|\tau_{i}-\tau_{j}\right|^{N-2}}-B_{N} \sum_{i=1}^{k} d_{i}^{2}\right]+\Theta,
\end{aligned}
$$

where

$$
A_{N}:=\frac{K_{N}^{-N}}{24 N(N-4)(N-6)}, B_{N}:=\frac{2(N-1) K_{N}^{-N}}{N(N-2)(N-4)}, D_{N}:=\frac{K_{N}^{-N}}{N}, E_{N}:=\alpha_{N} \int_{\mathbb{R}^{N}} U^{p}(y) d y
$$

and $K_{N}$ is the best constant for the embedding of $D^{1,2}\left(\mathbb{R}^{N}\right)$ into $L^{2^{*}}\left(\mathbb{R}^{N}\right)$. Here $\Theta$ is a smooth function such that $|\Theta|,|\nabla \Theta|=O\left(\varepsilon^{3 \frac{N-2}{N}+\zeta}\right)$ for some small $\zeta>0$.

Let us prove $(4.2)$.

$$
\begin{aligned}
J_{\varepsilon}\left(\sum_{i=1}^{k} \mathcal{W}_{i}\right)= & \underbrace{\sum_{i=1}^{k} J_{\varepsilon}\left(\mathcal{W}_{i}\right)}_{I}-\underbrace{\sum_{j<i} \int_{M} f\left(\mathcal{W}_{i}\right) \mathcal{W}_{j} d \nu_{g}}_{I I} \\
& +\sum_{i<j} \int_{M}\left[\nabla_{g} \mathcal{W}_{i} \nabla_{g} \mathcal{W}_{j}+\beta_{N} \mathrm{R}_{g} \mathcal{W}_{i} \mathcal{W}_{j}-f\left(\mathcal{W}_{i}\right) \mathcal{W}_{j}\right] d \nu_{g} \\
& -\int_{M}\left[F\left(\sum_{i=1}^{k} \mathcal{W}_{i}\right)-\sum_{i=1}^{k} F\left(\mathcal{W}_{i}\right)-\sum_{i \neq j} f\left(\mathcal{W}_{i}\right) \mathcal{W}_{j}\right] d \nu_{g}+\varepsilon \sum_{i<j} \int_{M} \mathcal{W}_{i} \mathcal{W}_{j} d \nu_{g} .
\end{aligned}
$$

First of all, we estimate the two leading terms $I$ and $I I$ in (4.4).

The term $I$ is given by the contribution of each bubble. Indeed, in Section 4 of [7] it was proved that for any $i=1, \ldots, k$

$$
J_{\varepsilon}\left(\mathcal{W}_{i}\right)=D_{N}-A_{N}\left|\operatorname{Weyl}_{g}\left(\xi_{i}\right)\right|_{g}^{2} \mu_{i}^{4}+\varepsilon B_{N} \mu_{i}^{2}+\left\{O\left(\varepsilon^{\frac{5}{2}}\right) \text { if } N=7, O\left(\varepsilon^{3}|\ln \varepsilon|^{3}\right) \text { if } N=8, O\left(\varepsilon^{3}\right) \text { if } N \geq 9\right\} \text {. }
$$

Now, by the choice of $d_{0}$ in (3.2) and the choice of $\mu_{i}, \alpha$ and $\beta$ in (3.4), we get

$$
\begin{aligned}
& \left|\operatorname{Weyl}_{g}\left(\xi_{i}\right)\right|^{2}=\left|\operatorname{Weyl}_{g}\left(\xi_{0}\right)\right|^{2}+\frac{1}{2} \mathcal{Q}\left(\xi_{0}\right)\left[\tau_{i}, \tau_{i}\right] \varepsilon^{2 \beta}+O\left(\varepsilon^{3 \beta}\right), \\
& \mu_{i}^{4}=\varepsilon^{4 \alpha}\left[d_{0}^{4}+4 d_{0}^{3} d_{i} \varepsilon^{\beta}+6 d_{0}^{2} d_{i}^{2} \varepsilon^{2 \beta}+O\left(\varepsilon^{3 \beta}\right)\right], \\
& \mu_{i}^{2}=\varepsilon^{2 \alpha}\left[d_{0}^{2}+2 d_{0} d_{i} \varepsilon^{\beta}+d_{i}^{2} \varepsilon^{2 \beta}\right] .
\end{aligned}
$$

Therefore, a straightforward computation shows that

$$
\begin{aligned}
-A_{N}\left|\operatorname{Weyl}_{g}\left(\xi_{i}\right)\right|_{g}^{2} \mu_{i}^{4}+\varepsilon B_{N} \mu_{i}^{2} & =\varepsilon^{2}\left[-A_{N}\left|\operatorname{Weyl}_{g}\left(\xi_{0}\right)\right|_{g}^{2}+B_{N} d_{0}^{2}\right] \\
& +\varepsilon^{3 \frac{N-2}{N}}\left[-\frac{1}{2} A_{N} d_{0}^{4} \mathcal{Q}\left(\xi_{0}\right)\left(\tau_{i}, \tau_{i}\right)-B_{N} \sum_{i=1}^{k} d_{i}^{2}\right]+O\left(\varepsilon^{\frac{7 N-18}{2 N}}\right) .
\end{aligned}
$$

By (4.5) and (4.6) we deduce the estimate of $I$.

The term $I I$ is given by the interaction of different bubbles. For any $h=1, \ldots, k$ let $B_{h}:=B\left(\varepsilon^{\beta} \tau_{h}, \varepsilon^{\beta} \sigma / 2\right)$. By (3.3) we deduce that $B_{h} \subset B\left(0, r_{0}\right)$ provided $\sigma$ is small enough and they are disjoint. Therefore, if 
$i \neq j$

$$
\begin{aligned}
\int_{M} f\left(\mathcal{W}_{i}\right) \mathcal{W}_{j} d \nu_{g} & =\int_{B_{i}} f\left(W_{i}(x)\right) W_{j}(x)|g(x)|^{1 / 2} d x+\int_{B\left(0, r_{0}\right) \backslash B_{i}} f\left(W_{i}(x)\right) W_{j}(x)|g(x)|^{1 / 2} d x \\
& +\int_{B\left(0, r_{0}\right)}\left[1-\chi^{p+1}(|x|)\right] f\left(W_{i}(x)\right) W_{j}(x)|g(x)|^{1 / 2} d x \\
& =E_{N} d_{0}^{N-2} \frac{1}{\left|\tau_{i}-\tau_{j}\right|^{N-2}} \varepsilon^{3 \frac{N-2}{N}}+O\left(\varepsilon^{3}\right) .
\end{aligned}
$$

Indeed, the main term of (4.7) is given by

$$
\begin{aligned}
& \int_{B_{i}} f\left(W_{i}(x)\right) W_{j}(x)|g(x)|^{1 / 2} d x \\
& =\int_{B_{i}} f\left(\mu_{i}^{-\frac{N-2}{2}} U\left(\frac{x-\varepsilon^{\beta} \tau_{i}}{\mu_{i}}\right)+\mu_{i}^{-\frac{N-6}{2}} \eta\left(\frac{x-\varepsilon^{\beta} \tau_{i}}{\mu_{i}}\right) V\left(\frac{x-\varepsilon^{\beta} \tau_{i}}{\mu_{i}}\right)\right) \times \\
& \times\left(\mu_{j}^{-\frac{N-2}{2}} U\left(\frac{x-\varepsilon^{\beta} \tau_{j}}{\mu_{j}}\right)+\mu_{i}^{-\frac{N-6}{2}} \eta\left(\frac{x-\varepsilon^{\beta} \tau_{j}}{\mu_{j}}\right) V\left(\frac{x-\varepsilon^{\beta} \tau_{j}}{\mu_{j}}\right)\right)|g(x)|^{1 / 2} d x \\
& \left(\eta\left(\frac{x-\varepsilon^{\beta} \tau_{j}}{\mu_{j}}\right)=0 \text { if } x \in B_{i} \text { and } \varepsilon\right. \text { is small enough) } \\
& =\int_{B_{i}} f\left(\mu_{i}^{-\frac{N-2}{2}} U\left(\frac{x-\varepsilon^{\beta} \tau_{i}}{\mu_{i}}\right)+\mu_{i}^{-\frac{N-6}{2}} \eta\left(\frac{x-\varepsilon^{\beta} \tau_{i}}{\mu_{i}}\right) V\left(\frac{x-\varepsilon^{\beta} \tau_{i}}{\mu_{i}}\right)\right) \times \\
& \times\left(\mu_{j}^{-\frac{N-2}{2}} U\left(\frac{x-\varepsilon^{\beta} \tau_{j}}{\mu_{j}}\right)\right)|g(x)|^{1 / 2} d x \\
& \text { (setting } x-\varepsilon^{\beta} \tau_{i}=\mu_{i} y \text { ) } \\
& =\mu_{i}^{\frac{N-2}{2}} \int_{B\left(0, \varepsilon^{\beta} \sigma / 2 \mu_{i}\right)} f\left(U(y)+\mu_{i}^{2} \eta(|y|) V(y)\right) \frac{\alpha_{N} \mu_{j}^{\frac{N-2}{2}}}{\left(\mu_{j}^{2}+\left|\mu_{j} y+\varepsilon^{\beta}\left(\tau_{i}-\tau_{j}\right)\right|^{2}\right)^{\frac{N-2}{2}} \times} \\
& \times\left|g\left(\mu_{i} y+\varepsilon^{\beta} \tau_{i}\right)\right|^{1 / 2} d y \\
& =\alpha_{N} \frac{\mu_{i}^{\frac{N-2}{2}} \mu_{j}^{\frac{N-2}{2}}}{\varepsilon^{\beta(N-2)}\left|\tau_{i}-\tau_{j}\right|^{N-2}}\left[\int_{\mathbb{R}^{N}} U^{p}(y) d y+O\left(\mu_{i}^{2}\right)+O\left(\left(\frac{\mu_{i}}{\varepsilon^{\beta}}\right)^{N}\right)\right] \\
& =\varepsilon^{3 \frac{N-2}{N}} \frac{d_{0}^{N-2}}{\left|\tau_{i}-\tau_{j}\right|^{N-2}} \alpha_{N} \int_{\mathbb{R}^{N}} U^{p}(y) d y+O\left(\varepsilon^{3}\right),
\end{aligned}
$$

because of the choice of $\mu_{i}$ in (3.4). Moreover, by (4.1)

$$
\begin{aligned}
& \left.\left|\int_{B\left(0, r_{0}\right) \backslash B_{i}} f\left(W_{i}(x)\right) W_{j}(x)\right| g(x)\right|^{1 / 2} d x \mid \\
& \left.\leq c \int_{\mathbb{R}^{N \backslash B_{i}}} \frac{\mu_{i}^{\frac{N+2}{2}}}{\left|x-\varepsilon^{\beta} \tau_{i}\right|^{N+2}} \frac{\mu_{j}^{\frac{N-2}{2}}}{\left|x-\varepsilon^{\beta} \tau_{j}\right|^{N-2}} d x \text { (setting } x=\varepsilon^{\beta} y\right) \\
& \leq c \frac{\mu_{i}^{\frac{N+2}{2}} \mu_{j}^{\frac{N-2}{2}}}{\beta^{N}} \int_{\mathbb{R}^{N} \backslash B\left(\tau_{i}, \sigma / 2\right)} \frac{1}{\left|y-\tau_{i}\right|^{N+2}} \frac{\mu_{j}^{\frac{N-2}{2}}}{\left|y-\tau_{j}\right|^{N-2}} d y \\
& =O\left(\frac{\mu_{i}^{\frac{N+2}{2}} \mu_{j}^{\frac{N-2}{2}}}{\varepsilon^{\beta N}}\right)=O\left(\varepsilon^{3}\right)
\end{aligned}
$$


and

$$
\left.\left|\int_{B\left(0, r_{0}\right)}\left[1-\chi^{p+1}(|x|)\right] f\left(W_{i}(x)\right) W_{j}(x)\right| g(x)\right|^{1 / 2} d x \mid=O\left(\varepsilon^{\frac{N}{2}}\right) .
$$

Finally, let us prove that all the other terms in (4.4) are of higher order.

By (2.5) and (2.6), we deduce that

$$
\left|\Delta_{g} \mathcal{W}_{i}+\beta_{N} \mathrm{R}_{g} \mathcal{W}_{i}-f\left(\mathcal{W}_{i}\right)\right|\left(\exp _{\xi_{0}}(x)\right) \leq c \frac{\mu_{i}^{\frac{N-2}{2}}}{\left(\mu_{i}^{2}+\left|x-\varepsilon^{\beta} \tau_{i}\right|^{2}\right)^{\frac{N-2}{2}}}
$$

and so by (4.1) if $i \neq j$ we have

$$
\begin{aligned}
& \left|\int_{M}\left[\nabla_{g} \mathcal{W}_{i} \nabla_{g} \mathcal{W}_{j}+\beta_{N} \mathrm{R}_{g} \mathcal{W}_{i} \mathcal{W}_{j}-f\left(\mathcal{W}_{i}\right) \mathcal{W}_{j}\right] d \nu_{g}\right| \\
& =\left|\int_{M}\left[\Delta_{g} \mathcal{W}_{i}+\beta_{N} \mathrm{R}_{g} \mathcal{W}_{i}-f\left(\mathcal{W}_{i}\right)\right] \mathcal{W}_{j} d \nu_{g}\right| \\
& \leq c \int_{B\left(0, r_{0}\right)} \frac{\mu_{i}^{\frac{N-2}{2}}}{\left|x-\varepsilon^{\beta} \tau_{i}\right|^{N-2}} \frac{\mu_{j}^{\frac{N-2}{2}}}{\left|x-\varepsilon^{\beta} \tau_{j}\right|^{N-2}} d x\left(\operatorname{setting} x=\varepsilon^{\beta} y\right) \\
& \leq c \frac{\mu_{i}^{\frac{N-2}{2}}}{\mu_{j}^{\frac{N-2}{2}}} \int_{\varepsilon^{\beta N-4}} \frac{1}{\left|y-\tau_{i}\right|^{N-2}} \frac{1}{\left|y-\tau_{j}\right|^{N-2}} d x=O\left(\varepsilon^{3}\right) .
\end{aligned}
$$

Moreover, if $i \neq j$

$$
\begin{aligned}
& \left|\int_{M} \mathcal{W}_{i} \mathcal{W}_{j} d \nu_{g}\right|=\left.\left|\int_{B\left(0, r_{0}\right)} W_{i}(x) W_{j}(x)\right| g(x)\right|^{1 / 2} d x \mid \\
& \left.\leq c \int_{B\left(0, r_{0}\right)} \frac{\mu_{i}^{\frac{N-2}{2}}}{\left|x-\varepsilon^{\beta} \tau_{i}\right|^{N-2}} \frac{\mu_{j}^{\frac{N-2}{2}}}{\left|x-\varepsilon^{\beta} \tau_{j}\right|^{N-2}} d x \text { (setting } x=\varepsilon^{\beta} y\right) \\
& \leq c \frac{\mu_{i}^{\frac{N-2}{2}} \mu_{j}^{\frac{N-2}{2}}}{\varepsilon^{\beta N-4}} \int_{\mathbb{R}^{N}} \frac{1}{\left|y-\tau_{i}\right|^{N-2}} \frac{1}{\left|y-\tau_{j}\right|^{N-2}} d x=O\left(\varepsilon^{3}\right) .
\end{aligned}
$$

Finally, we have

$$
\begin{aligned}
& \int_{M}\left[F\left(\sum_{i=1}^{k} \mathcal{W}_{i}\right)-\sum_{i=1}^{k} F\left(\mathcal{W}_{i}\right)-\sum_{i \neq j} f\left(\mathcal{W}_{i}\right) \mathcal{W}_{j}\right] d \nu_{g} \\
& =\sum_{h=1}^{k} \int_{B_{h}}\left[F\left(\sum_{i=1}^{k} W_{i}\right)-\sum_{i=1}^{k} F\left(W_{i}\right)-\sum_{i \neq j} f\left(W_{i}\right) W_{j}\right]|g(x)|^{1 / 2} d x \\
& +\int_{B\left(0, r_{0}\right) \backslash \cup_{h} B_{h}}\left[F\left(\sum_{i=1}^{k} W_{i}\right)-\sum_{i=1}^{k} F\left(W_{i}\right)-\sum_{i \neq j} f\left(W_{i}\right) W_{j}\right]|g(x)|^{1 / 2} d x \\
& +\int_{B\left(0, r_{0}\right)}\left[1-\chi^{p+1}(|x|)\right]\left[F\left(\sum_{i=1}^{k} W_{i}\right)-\sum_{i=1}^{k} F\left(W_{i}\right)-\sum_{i \neq j} f\left(W_{i}\right) W_{j}\right]|g(x)|^{1 / 2} d x .
\end{aligned}
$$


It is immediate that

$$
\int_{B\left(0, r_{0}\right)}\left[1-\chi^{p+1}(|x|)\right]\left[F\left(\sum_{i=1}^{k} W_{i}\right)-\sum_{i=1}^{k} F\left(W_{i}\right)-\sum_{i \neq j} f\left(W_{i}\right) W_{j}\right]|g(x)|^{1 / 2} d x=O\left(\varepsilon^{\frac{N}{2}}\right) .
$$

Moreover, outside the $k$ balls we get

$$
\begin{aligned}
& \int_{B\left(0, r_{0}\right) \backslash \cup_{h} B_{h}}\left|F\left(\sum_{i=1}^{k} W_{i}\right)-\sum_{i=1}^{k} F\left(W_{i}\right)-\sum_{i \neq j} f\left(W_{i}\right) W_{j}\right||g(x)|^{1 / 2} d x \\
& \leq c \sum_{i \neq j} \int_{B\left(0, r_{0}\right) \backslash \cup_{h} B_{h}}\left(\left|W_{i}\right|^{p-1} W_{j}^{2}+\left|W_{j}\right|^{p-1} W_{i}^{2}\right) d x=O\left(\varepsilon^{3}\right),
\end{aligned}
$$

because if $2<q=p+1<3$

$$
\left|(a+b)^{q}-a^{q}-b^{q}-q a^{q-1} b-q a b^{q-1}\right| \leq c\left(a^{2} b^{q-2}+a^{q-2} b^{2}\right) \text { for any } a, b>0
$$

and if $j \neq i$

$$
\begin{aligned}
& \left.\int_{B\left(0, r_{0}\right) \backslash \cup_{h} B_{h}}\left|W_{i}\right|^{p-1} W_{j}^{2} d x \leq c \int_{B\left(0, r_{0}\right) \backslash \cup_{h} B_{h}} \frac{\mu_{i}^{2}}{\left|x-\varepsilon^{\beta} \tau_{i}\right|^{4}} \frac{\mu_{j}^{N-2}}{\left|x-\varepsilon^{\beta} \tau_{j}\right|^{2(N-2)}} d x \text { (setting } x=\varepsilon^{\beta} y\right) \\
& \leq c \frac{\mu_{i}^{2} \mu_{j}^{N-2}}{\varepsilon^{\beta N}} \int_{\mathbb{R}^{N} \backslash \cup_{h} B\left(\tau_{h}, \sigma / 2\right)} \frac{1}{\left|y-\tau_{i}\right|^{4}} \frac{1}{\left|y-\tau_{j}\right|^{2(N-2)}} d x .
\end{aligned}
$$

On each ball $B_{h}$ we also have

$$
\begin{aligned}
& \int_{B_{h}}\left|F\left(\sum_{i=1}^{k} W_{i}\right)-\sum_{i=1}^{k} F\left(W_{i}\right)-\sum_{i \neq j} f\left(W_{i}\right) W_{j}\right| \mid g(x) \|^{1 / 2} d x \\
& \leq \int_{B_{h}}\left|F\left(W_{h}+\sum_{i \neq h} W_{i}\right)-F\left(W_{h}\right)-\sum_{j \neq h} f\left(W_{h}\right) W_{j}\right| d x \\
& +\sum_{i \neq h} \int_{B_{h}}\left|F\left(W_{i}\right)\right| d x+\sum_{\substack{i \neq h \\
j \neq i}} \int_{B_{h}}\left|f\left(W_{i}\right) W_{j}\right| d x \\
& \leq c \sum_{i \neq h} \int_{B_{h}} W_{h}^{p-1} W_{i}^{2} d x+c \sum_{i \neq h} \int_{B_{h}} W_{i}^{p+1} d x+c \sum_{\substack{i \neq h \\
j \neq i}} \int_{B_{h}} W_{i}^{p} W_{j} d x,
\end{aligned}
$$

because if $q=p+1 \geq 1$

$$
\left|(a+b)^{q}-a^{q}-q a^{q-1} b\right| \leq c\left(b^{q}+a^{q-2} b^{2}\right) \text { for any } a, b>0 .
$$

Now we use (4.1) and we get if $i \neq h$

$$
\begin{aligned}
& \int_{B_{h}} W_{h}^{p-1} W_{i}^{2} d x \leq c \int_{B_{h}} \frac{\mu_{h}^{2}}{\left|x-\varepsilon^{\beta} \tau_{h}\right|^{4}} \frac{\mu_{i}^{N-2}}{\left|x-\varepsilon^{\beta} \tau_{i}\right|^{2(N-2)}} d x\left(\text { setting } x=\varepsilon^{\beta} y\right) \\
& \leq c \frac{\mu_{h}^{2} \mu_{i}^{N-2}}{\varepsilon^{\beta N}} \int_{B\left(\tau_{h}, \sigma / 2\right)} \frac{1}{\left|y-\tau_{h}\right|^{4}} \frac{1}{\left|y-\tau_{i}\right|^{2(N-2)}} d y=O\left(\varepsilon^{3}\right),
\end{aligned}
$$

if $j, i \neq h$

$$
\begin{aligned}
& \int_{B_{h}}\left|W_{i}\right|^{p} W_{j} d x \leq c \int_{B_{h}} \frac{\mu_{i}^{\frac{N+2}{2}}}{\left|x-\varepsilon^{\beta} \tau_{i}\right|^{N+2}} \frac{\mu_{j}^{\frac{N-2}{2}}}{\left|x-\varepsilon^{\beta} \tau_{j}\right|^{N-2}} d x \\
& \leq c \frac{\mu_{i}^{\frac{N+2}{2}} \mu_{j}^{\frac{N-2}{2}}}{\varepsilon^{2 \beta N}}\left|B_{h}\right| \leq c \frac{\mu_{i}^{\frac{N+2}{2}} \mu_{j}^{\frac{N-2}{2}}}{\varepsilon^{\beta N}}=O\left(\varepsilon^{3}\right),
\end{aligned}
$$


if $i \neq h$

$$
\begin{aligned}
& \int_{B_{h}}\left|W_{i}\right|^{p} W_{h} d x \leq c \int_{B_{h}} \frac{\mu_{i}^{\frac{N+2}{2}}}{\left|x-\varepsilon^{\beta} \tau_{i}\right|^{N+2}} \frac{\mu_{h}^{\frac{N-2}{2}}}{\left|x-\varepsilon^{\beta} \tau_{h}\right|^{N-2}} d x \\
& \leq c \frac{\mu_{i}^{\frac{N-2}{2}}}{\varepsilon^{\beta(N+2)}} \int_{B_{h}} \frac{1}{\left|x-\varepsilon^{\beta} \tau_{h}\right|^{N-2}} d x \leq c \frac{\mu_{i}^{\frac{N-2}{2}} \mu_{h}^{\frac{N-2}{2}}}{\varepsilon^{\beta N}}=O\left(\varepsilon^{3}\right)
\end{aligned}
$$

and if $i \neq h$

$$
\int_{B_{h}} W_{i}^{p+1} d x \leq c \int_{B_{h}} \frac{\mu_{i}^{N}}{\left|x-\varepsilon^{\beta} \tau_{i}\right|^{2 N}} d x \leq c \frac{\mu_{i}^{N}}{\varepsilon^{2 \beta N}}\left|B_{h}\right| \leq c \frac{\mu_{i}^{N}}{\varepsilon^{\beta N}}=O\left(\varepsilon^{3}\right) .
$$

That concludes the proof.

\section{REFERENCES}

[1] T. Aubin Equations différentielles non linéaires et problème de Yamabe concernant la courbure scalaire, J. Math. Pures Appl. (9) (1976) 269-296

[2] A. Bahri Proof of the Yamabe conjecture, without the positive mass theorem, for locally conformally flat manifolds. Einstein metrics and Yang-Mills connections (Sanda, 1990), 126, Lecture Notes in Pure and Appl. Math., 145, Dekker, New York, 1993.

[3] G. Bianchi and H. Egnell A note on the Sobolev inequality, J. Funct. Anal. 100 (1991) 1 18-24

[4] S. Brendle Blow-up phenomena for the Yamabe equation, J. Amer. Math. Soc. 21 (2008), no. 4, 951979.

[5] S. Brendle and F.C. Marques Blow-up phenomena for the Yamabe equation. II, J. Differential Geom. 81 (2009), no. 2, 225250.

[6] O. Druet Compactness for Yamabe metrics in low dimensions, Int. Math. Res. Not. 23 (2004), 11431191.

[7] P. Esposito and A. Pistoia Blowing-up solutions for the Yamabe equation, Port. Math. 71 (2014), no. 3-4, 249-276

[8] P. Esposito, A. Pistoia, and J. Vtois The effect of linear perturbations on the Yamabe problem, Math. Ann. 358 (2014), no. $12,511-560$.

[9] M.A. Khuri, F.C. Marques, and R.M. Schoen A compactness theorem for the Yamabe problem, J. Differential Geom. 81 (2009), no. 1, 143-196

[10] J.M. Lee and T.H. Parker, The Yamabe problem, Bull. Amer. Math. Soc. (N.S.) 17 (1987), no. $1,3791$.

[11] Y.Y. Li and L. Zhang Compactness of solutions to the Yamabe problem. III, J. Funct. Anal. 245 (2007), no. 2, $438-474$.

[12] Y.Y. Li and M.J. Zhu Yamabe type equations on three-dimensional Riemannian manifolds, Commun. Contemp. Math. 1 (1999), no. 1, 150.

[13] F.C. Marques, A priori estimates for the Yamabe problem in the non-locally conformally flat case, J. Differential Geom. 71 (2005), no. 2, 315346.

[14] F. Morabito, A. Pistoia, G. Vaira in preparation

[15] M. Obata, The conjectures on conformal transformations of Riemannian manifolds, J. Differential Geom. 6 (1972) 247258 ,

[16] D. Pollack, Nonuniqueness and high energy solutions for a conformally invariant scalar curvature equation, Comm. Anal. and Geom. 1 (1993) 347-414,

[17] A. Pistoia, G. Vaira From periodic ODE's to supercritical PDE's. Nonlinear Anal. 119 (2015), 330340.

[18] F. Robert and J. Vétois Examples of non-isolated blow-up for perturbations of the scalar curvature equation on nonlocally conformally flat manifolds, J. Differential Geom. 98 (2014), no. 2, 349356.

[19] F. Robert and J. Vétois A general theorem for the construction of blowing-up solutions to some elliptic nonlinear equations via Lyapunov-Schmidt's reduction, Concentration Compactness and Profile Decomposition (Bangalore, 2011), Trends in Mathematics, Springer, Basel, 2014, pp. 85116.

[20] R. Schoen Conformal deformation of a Riemannian metric to constant scalar curvature, J. Differential Geometry 20 (1984) 479-495,

[21] R. Schoen Variational theory for the total scalar curvature functional for Riemann-ian metrics and related topics, in 'Topics in Calculus of Variations,' Lecture Notes in Mathematics, Springer-Verlag, New York, 1365, 1989,

[22] R.M. Schoen, Notes from graduates lecture in Stanford University http://www.math.washington.edu/pollack/research/Schoen-1988-notes.html.

[23] R.M. Schoen On the number of constant scalar curvature metrics in a conformal class, Differential geometry, Pitman Monogr. Surveys Pure Appl. Math., 52, Longman Sci. Tech., Harlow, 1991, 311-320

[24] R.M. Schoen and D. Zhang, Prescribed scalar curvature on the $n$-sphere, Calc. Var. and PDEs 4 (1996) 1-25

[25] N.S. Trudinger, Remarks concerning the conformal deformation of Riemannian structures on compact manifolds, Ann. Scuola Norm. Sup. Pisa (3) (1968) 265-274

[26] H. Yamabe On a deformation of Riemannian structures on compact manifolds Osaka Math. J. 12 (1960) 21-37 
Angela Pistoia, Dipartimento di Scienze di Base e Applicate Per l'Ingegneria, Sapienza Università di Roma, via Antonio Scarpa 16, 00161 Roma, Italy

E-mail address: angela.pistoia@uniroma1.it

Giusi Vaira, Dipartimento di Scienze di Base e Applicate per l'Ingegneria, Sapienza Università di Roma, via Antonio Scarpa 16, 00161 Roma, Italy

E-mail address: vaira.giusi@gmail.com 